\title{
INTELLIGENT REVERSE-ENGINEERING SEGMENTATION: AUTOMATIC SEMANTIC RECOGNITION OF LARGE 3D DIGITALIZED CLOUD OF POINTS DEDICATED TO HERITAGE OBJECTS
}

\author{
Florent Laroche \\ LUNAM Université, \\ Ecole Centrale Nantes, \\ IRCCYN UMR CNRS 6597, \\ 1 , rue de la Noë, BP 92101, \\ 44321 Nantes Cedex 3, France \\ Benjamin Hervy \\ LUNAM Université, \\ Ecole Centrale Nantes, \\ IRCCYN UMR CNRS 6597, \\ 1 , rue de la Noë, BP 92101, \\ 44321 Nantes Cedex 3, France
}

\author{
Daniel Lefèvre \\ LUNAM Université, \\ Ecole Centrale Nantes, \\ 1, rue de la Noë, BP 92101, \\ 44321 Nantes Cedex 3, \\ France
}

\author{
Myriam Servières \\ LUNAM Université, \\ Ecole Centrale Nantes, \\ CERMA-IRSTV UMR CNRS 1563, \\ 1, rue de la Noë, BP 92101, \\ 44321 Nantes Cedex 3, France
}

\author{
Alain Bernard \\ LUNAM Université, \\ Ecole Centrale Nantes, \\ IRCCYN UMR CNRS 6597, \\ 1 , rue de la Noë, BP 92101, \\ 44321 Nantes Cedex 3, France
}

\section{ABSTRACT}

In this article we present a multidisciplinary experimentation realized between a mechanical laboratory, a computer scientist laboratory and a museum.

Our goal is to provide automatic tools for non-expert people who want to use 3D digitized elements. After scanning an objet, we obtain a huge amount of points. In order to manipulate it, it is necessary to decimate it. However, when doing this operation, we can optimize the algorithms for creating semantic topology; obviously we can do it automatically. Consequently, we are going to do what we name segmentation: we extract meaning from $3 \mathrm{D}$ points and meshes.

Our experimentation deals with a physical mock-up of Nantes city that have been designed in 1900. After digitalization, we have created a software that can:

1. use the whole 3D cloud of points as an input;

2. fill a knowledge database with an intelligent segmentation of the 3D virtual models: ground, walls, roofs...

This use case is the first step of our research. At the end, we aim to deploy our method to complex mechanical parts. Nowadays, when designing CAD parts we use as well as volume parts than surface parts or meshes. We know is it not necessary to reconstruct all the triangles. It is a lost of time and we can directly use cloud of points for CAD design. However, the design tree will not be updated. So, with our method, imagine that one day we can digitalize a motor and a system could automatically create the 3D mock-up and the design tree.

\section{KEYWORDS}

Reverse-engineering, 3D digitalization, semantic recognition, museums, Advanced Industrial Archaeology.

\section{INTRODUCTION}

In Museum, many testimonies of our cultural heritage can be found. Nevertheless because of their high fragility, public cannot get too close to them. This communication will detail one experiment we are conducting with a French museum. The considered object is a physical mock-up of Nantes harbor; the mock-up has been built in 1899 and shown in 1900 during the World's Fair that took place in Paris, France [1]. But nowadays, this object is shown behind panes at the "Château des Ducs" museum in Nantes, France. The object has been digitized and one 3D model has been reconstructed. Thanks to a virtual system coupled to a touchscreen with semantic research modules and to a light pointer, it will allow the visitor to better understand the mock-up and the associated history. Moreover, the system will be user-centered: the visitor will interact directly with the digital mock-up and at the same time with all the knowledge brought by contextualization. 
Our proposal consists of a new reverse-engineering method with automatic semantic recognition. From a technological point of view, there are several steps to go from cloud of points to $3 \mathrm{D}$ virtual mock-up. We aim to realize those operations automatically because of the large amount of triangles we have obtained with the 3D digitalization. Nowadays, there are no technologies that are able to load and visualize easily more than $250,000,000$ points. So, first we get a huge cloud of points; but the acquisition is not perfect: there are some holes in the mesh. After filling them, instead of decimating manually the 3D cloud of points, we are going to decimate it automatically. This step allows cutting the 3D model into multiples parts that have semantic meaning. Indeed, it is what we called the vectorization step. It consists of a 3D model simplification keeping the topology of the $3 \mathrm{D}$ cloud of points. In order to do so, we spread the $3 \mathrm{D}$ vectorized mock-up into multiple semantic parts. We segment it into walls and roofs of buildings, grounds and other objects (boats) and zones (water of the river). The segmentation phase is important because it represents the beginning of creating an intelligent 3D mock-up. Consequently, it allows creating a semantic link between the $3 \mathrm{D}$ virtual mock-up and data into the knowledge database (like photos, maps and geographical coordinates). [19]

\section{STATE OF THE ART}

Nantes 1900 project is not the only one dealing with mock-up digitalization and 3D object simplification. We will start by introducing other mock-up digitalization projects then we will present some known algorithms used to simplify a high detailed mesh.

We can find in the literature few projects that propose to digitize a large heritage object and create a virtual model with many levels of details that are chosen by the users [2-6].

An experiment has been done by the "Musée des Maquettes" in France [3]. The mock-up representing the city of Toul was digitalized in 3D. Then, the clouds of points have been combined in order to obtain a parametric virtual model. In the 3D digitalization of a physical mock-up of the city of Prague designed by Langweil, data have been acquired by photographic systems [2]. Then, the clouds of points have been combined in order to obtain a parametric virtual model.

Rome Reborn is a project in the same field. It aims to rebuild the city of Rome between the years $1000 \mathrm{BC}$ to $500 \mathrm{AD}$. This project required numerous plans but also historical documents to understand the physical mock-up [4-6].

Last experimentation known is the $3 \mathrm{D}$ digitalization of a physical mock-up of the city center of Liège where the acquisition was performed using an interferometric system [13]. This experimentation is probably the most advanced use case done in parallel with our project. Indeed, the multidisciplinary team also refuses to realize each step manually. We are currently comparing our methods in order to reach to common guidelines.
Every kind of digitization raised numerous problems. One of the most important is that it is impossible to visualize the enormous quantity of points acquired: files are so heavy. There are no software that can manipulate easily 200 millions of points. Consequently, digitized data were divided into several areas for being viewed independently and then simplified. We can find similar projects with art works digitization problem as The Digital Michelangelo Project [7]. However, this segmentation method does not fit with our heritage object due to the fact that dividing large-scale data into sub-regions will lead to lose some information. Indeed, during the digitalization phase, triangles and their orientations have been captured. If we cut the large-scale mesh and simplify small parts, it would be necessary to sew them if we want to obtain the whole mock-up.

Contrary to examples presented in this state of the art, the specificity of all those projects is that the work is done manually; nothing is automatic [10]. Few algorithms are presented but they only state the process followed [12]. Concerning our project named "Nantes1900 project", it would be impossible to do it manually due to the amount of data: neither the 3D cloud of points, nor the historical documents that can help to enrich and understand the 3D mock-up [11]. Then we choose to simplify automatically the $3 \mathrm{D}$ mesh. In computer graphic domain, a lot of algorithms exist for segmenting and then simplifying meshes, for example to obtain a real time rendering of complexes scenes.

The mesh segmentation is a research field of its own and is the first step of the process before a mesh simplification. Interested reader can find surveys of these methods in [14] and [15]. Shamir has classified the possible approximate solutions for mesh segmentation according to the approaches as: region growing, hierarchical clustering, iterative clustering, spectral analysis and implicit methods [14]. Our method can be classified as an implicit one based on difference in normal of vertices and angles between faces, but it is much simpler than those described in [14] as explained in the PROCESS AND RESULTS section.

It exists several algorithms for simplifying meshes, some of them are created for mesh optimization, and others are point based rendering. We use meshes and not cloud of points because as triangles have been digitized we can use their orientation to compute better algorithms. The mesh simplification techniques optimize the display of objects made of a very large number of polygons in reducing their number. Those techniques are used when the viewed object is far from the camera and fine details of its geometry is not apparent or do not require to be displayed. Consequently, each simplified version of the global mock-up will display various levels of details. Those algorithms can produce meshes with a topology that is quasi-identical to the original one. It will depend on the simplification aim, get the fixed number of final meshes and/or minimize the error. [16] details the algorithms giving a discrete set of simplified versions of a given complex mesh. Other 
algorithms, as progressive meshes [17], are using edges collapse to simplify the meshes.

Now it exists a technique to display a large number of acquisition point in real time, considering only point and not meshes [17]. This technique was based on multiresolution hierarchy [21] based on bounding spheres with a rendering system based on points and was developed for display purpose [20]. We did not follow it because our purpose was to analyze the data rather than display it. Indeed, the final step of the automatic segmentation we are developing is to create automatically the structure of a knowledge database.

\section{PROCESS AND RESULTS}

The general process can be synthesized in two steps as shown by figure 1 (steps $\mathrm{A} 1$ and $\mathrm{A} 2$ of the $\mathrm{A} 0$ diagram):

1. $3 \mathrm{D}$ acquisition and process

2. Semantic conversion.

The first process transforms the acquired physical mock-up into an unrefined mesh (A1). Nevertheless, this part takes the most time. The second one (A2) transforms these unrefined meshes into a simplified (nearly vectorized) mock-up with semantic information.

\subsection{D ACQUISITION AND PROCESS}

The first step is the 3D scan that converts the physical object into a digital object (process shown on figure 2 ). The physical mock-up was scanned with a VIUscan from the company Creaform. The scale of the obtained mesh is about
$1 / 450$. But it is not perfect: in our case, a lot of objects are not correctly scanned (little houses, courtyards, etc.). Moreover, the scan is divided into many pieces, and it need to be merged (see figure 3). This step is realized routinely because we have digitalized all the pieces in the same reference. As there are overlapping, the merge does not require human intervention.
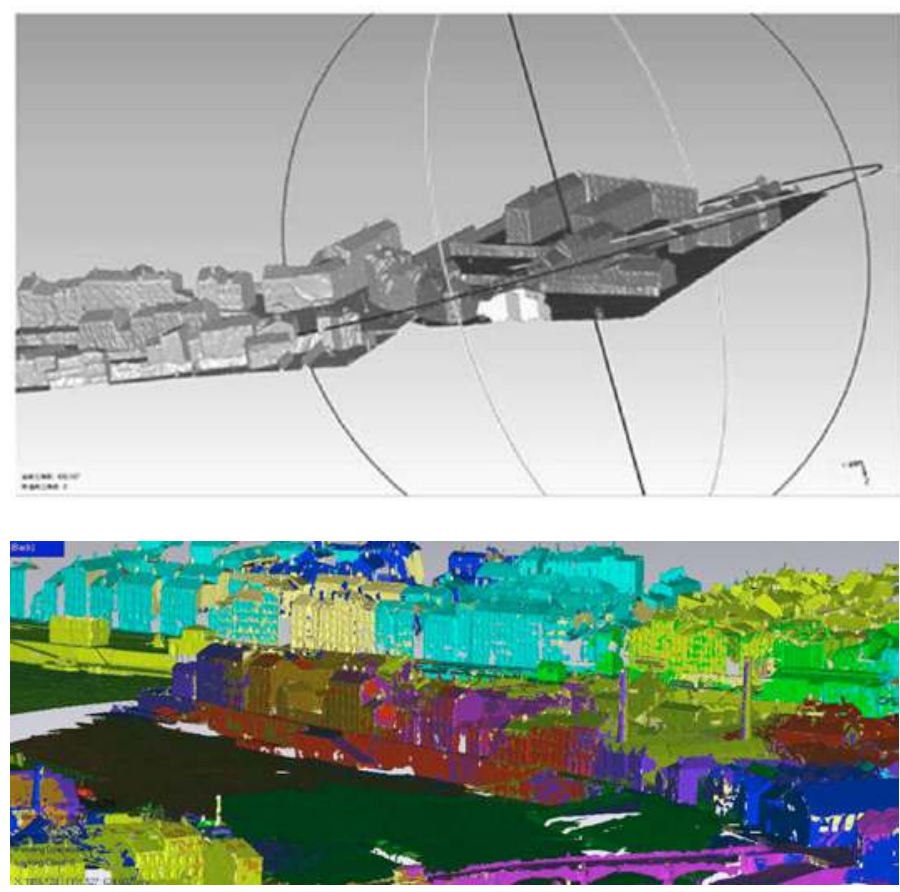

fig 3: Merging meshes

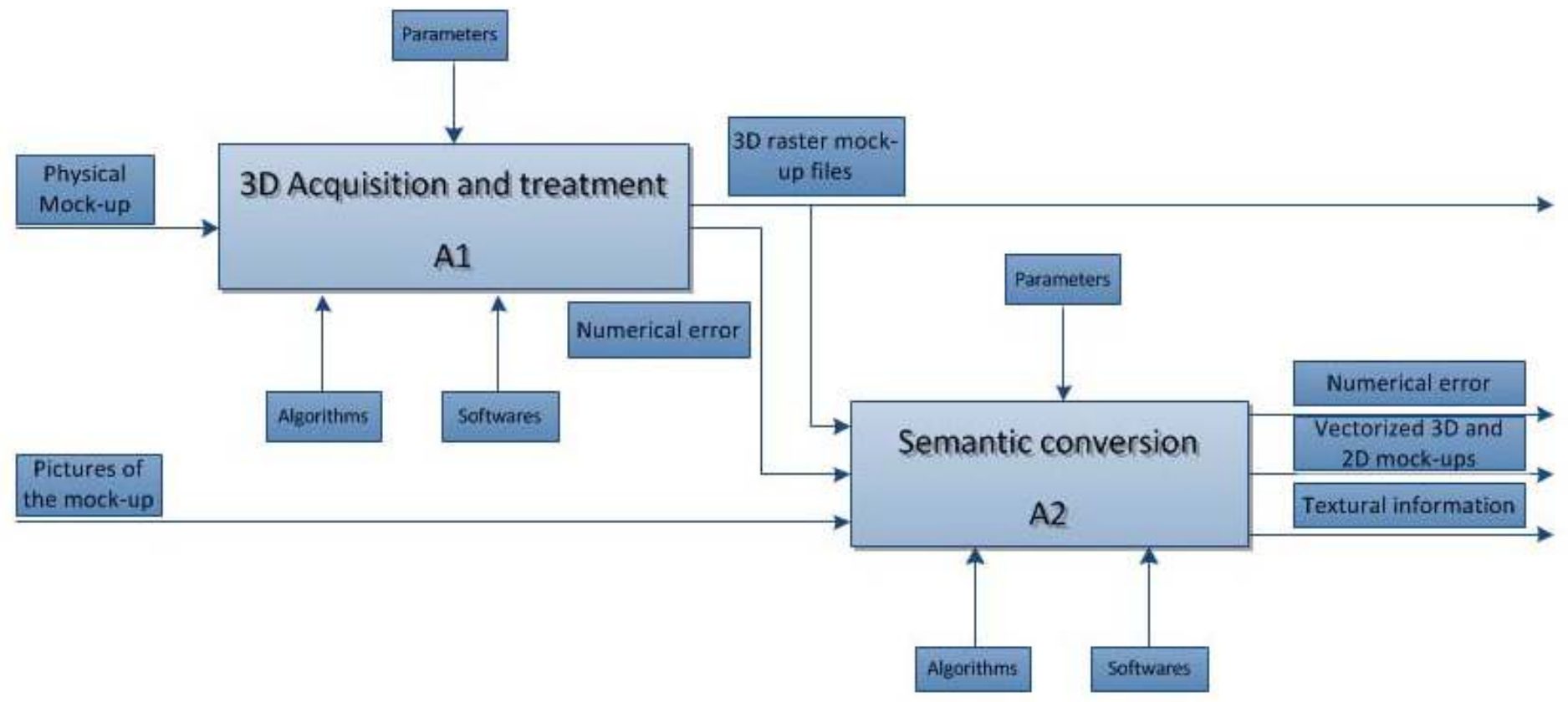

fig 1: SADT A0 diagram of our global process: the global method (A1 and A2) 


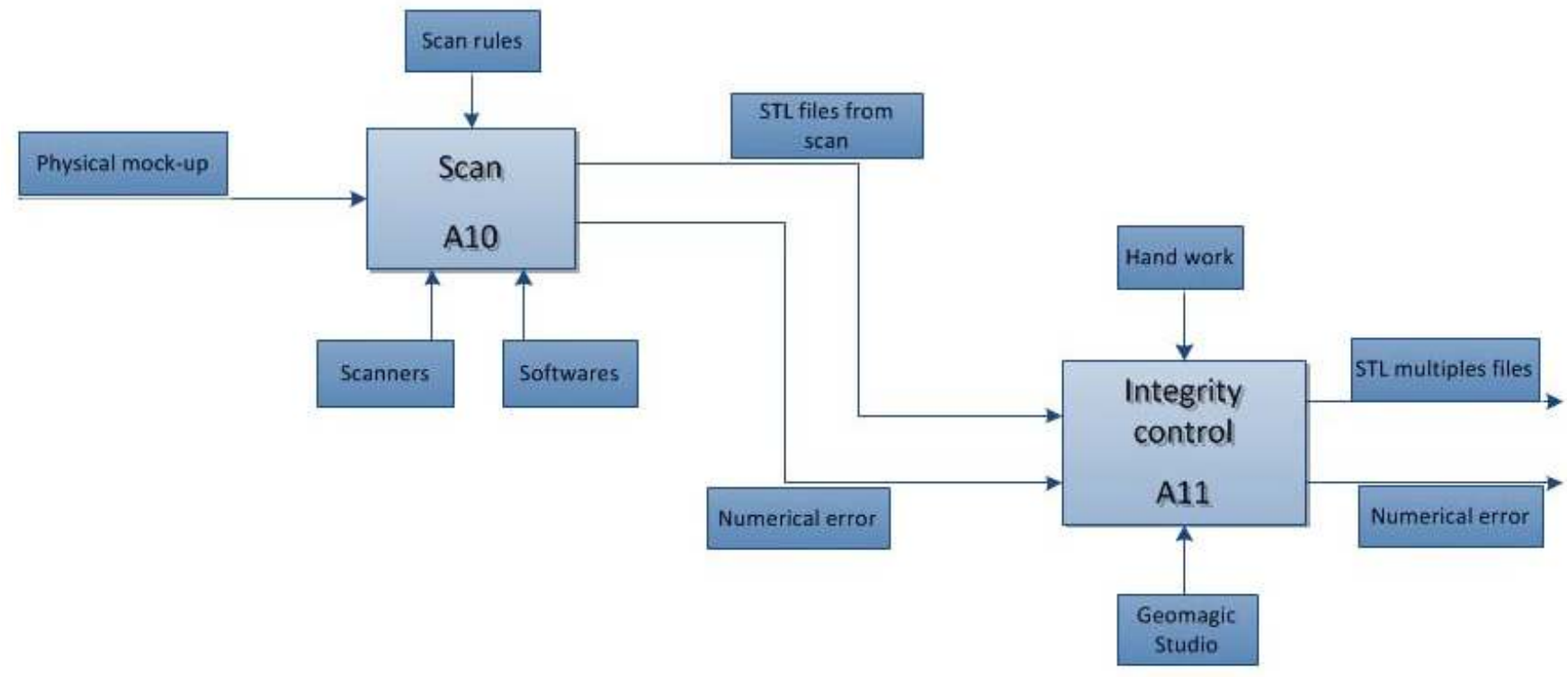

fig 2: SADT A1 diagram: 3D acquisition (A10) and integrity control (A11) method

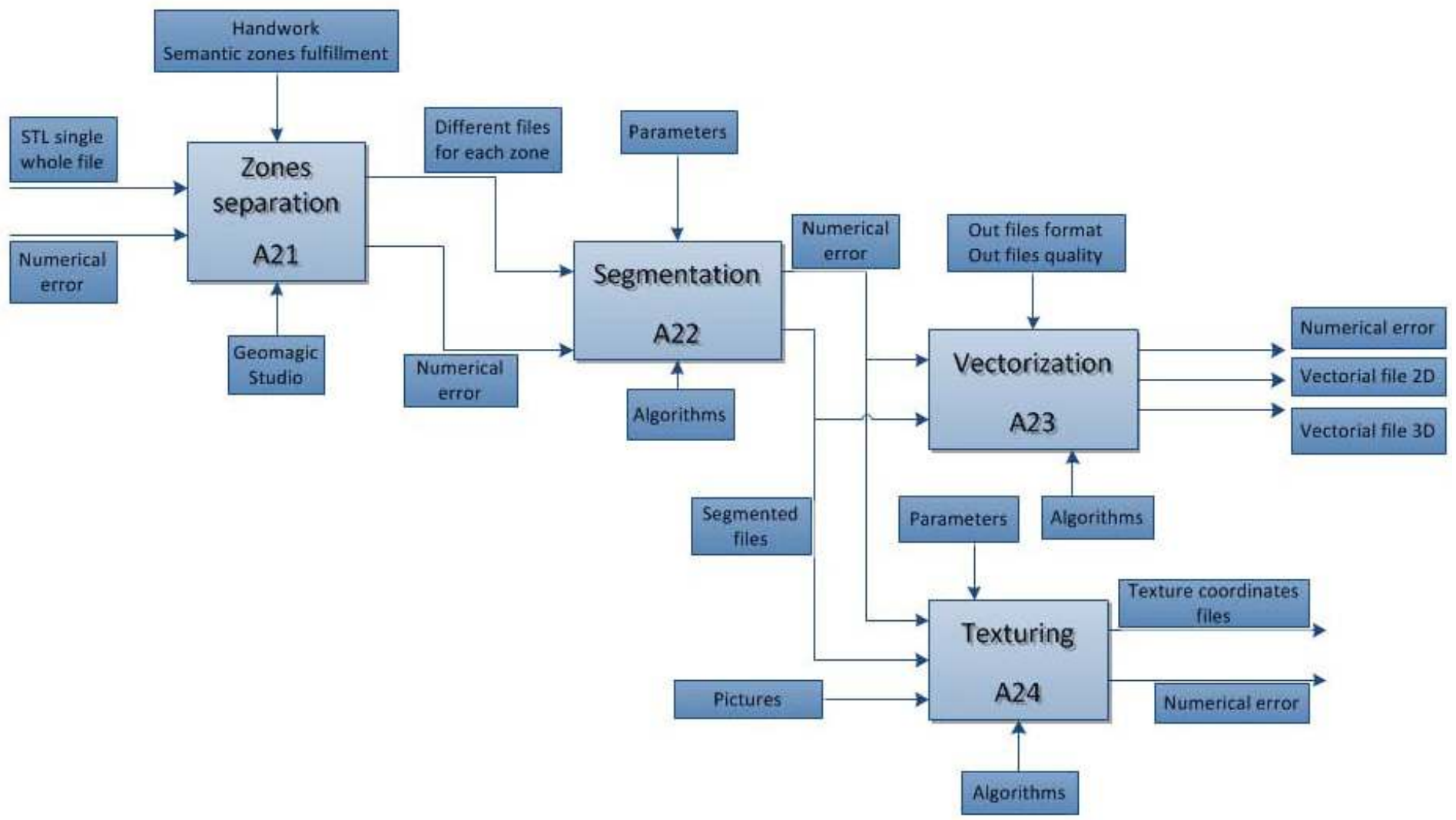

fig 5: SADT A2 diagram: zoning (A21), segmentation (A22) and vectorization (A23) 
After merging, the mesh that is obtained needs to be corrected. This integrity control is the pre-processing part (see example in figure 4). This step consists in finding errors (mainly missing parts) and correcting them. Those errors can be:

- Minor holes: few triangles are missing and it can be easily completed using some software,

- Middle holes: it needs more time, but it can be completed, considering the topology of the building and the pictures from the physical mock-up,

- Important holes: entire buildings are missing. They are very time consuming and maybe require a new scan,

- Cracks: as the physical mock-up is older and cracked, two versions of the mock-up have to be design: one corresponding exactly to the historical object, with the cracks (similar to earthquakes), and one without, to have the correct mesh representing the town,

- Little objects: trees, cranes, tramways and other little objects have not been correctly scanned because of their small size. Now, they can be reconstituted and added to the mesh.

This pre-processing part is very long because of the number of problems in the mock-up and due to the fact it is done quasi-manually. Indeed, the process is the following: after having established a zone with common characteristics, a specific construction algorithm is applied. Remark that this step is crucial for the following process algorithms and we did it very carefully.
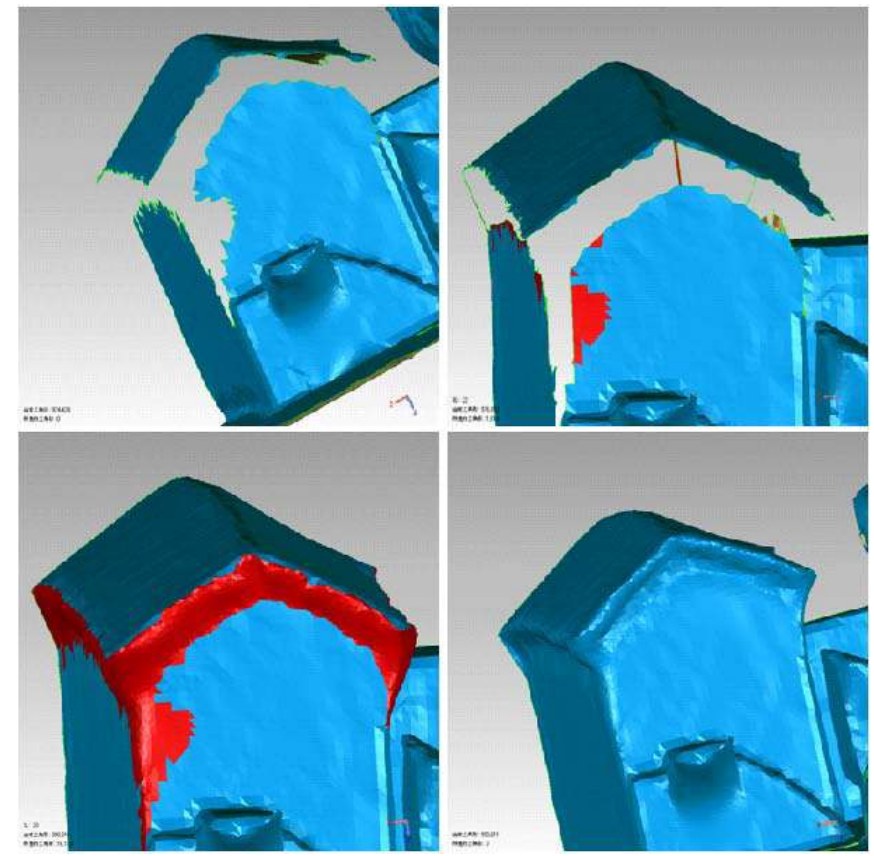

fig 4: example of holes filling

\subsection{SEMANTIC CONVERSION}

This step consists in adding information into the initial mesh, and, by the way, simplifying it in order to be able to manipulate it easily (see figure 5 for Semantic algorithm). We must remind that the initial mesh is composed of $250,000,000$ triangles.

Processing the whole mock-up is not possible; first, digital object has to be split into semantic zone or islet. There are 5 possible zones: residential, industrial, grounds, aqueous and others. Indeed, in the future, we will be able to apply similar algorithms to the similar buildings or neighborhood. The second goal of this step is to have a reasonable size for the next step of the process. The size is about 500000 triangles for one islet.

The main step of the semantic recognition consists of cutting the initial mesh. Indeed, if we pick two triangles in the mesh, we know everything about their location in $3 \mathrm{D}$, but we have absolutely no idea of their common relations. Do they belong to the same wall? building?; or perhaps triangles are independents: do they belong to a roof? a door? a chimney?. Next objective consists in cutting this new mesh: algorithms have been created and developed using Java language. The principle lies to the use of the orientation and the location of the triangles.

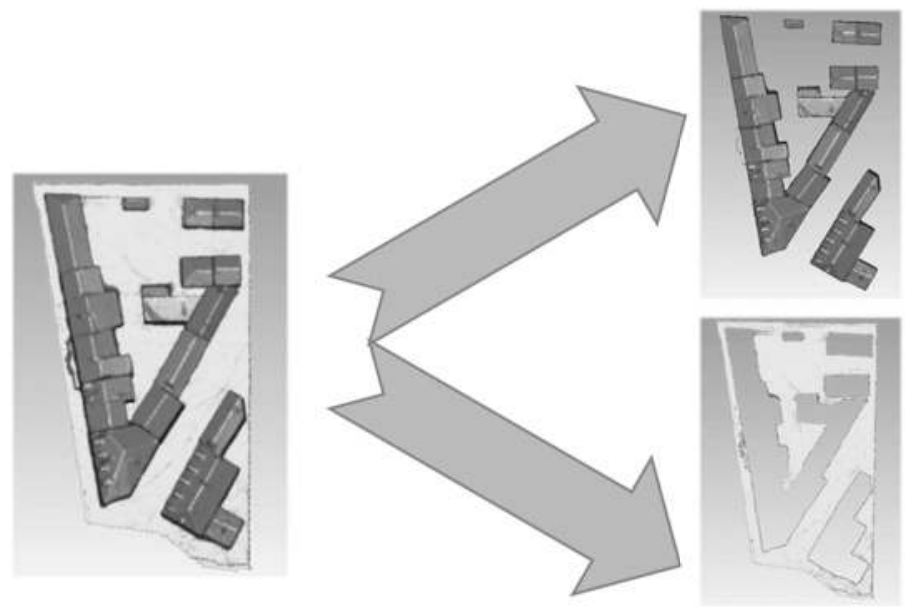

fig 6: Ground extraction result

The first step consists in extracting the ground (see figure 6): the direction of the ground of the current islet is given. The first idea was to extract the triangles oriented in this direction using the normal of those triangles. But, of course, some mistakes happened: few top of some roofs were picked and few other horizontal roofs were mingled with grounds. Then two coefficients are added:

- a vertical one, each ground must be located lower than a percentage of the size of the building,

- a size one, each ground must contain at least $\mathrm{X}$ triangles.

The algorithm is given in figure 7. 


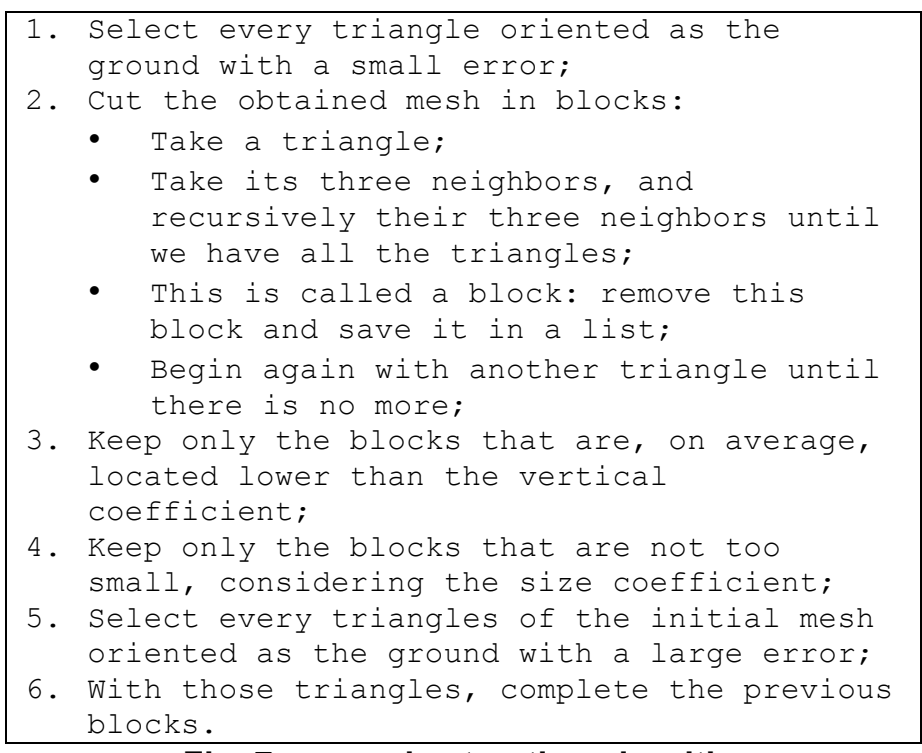

Fig. 7: ground extraction algorithm

After extracting the grounds, buildings remain. After cutting them into blocks, blocks are sorted and only the biggest ones are kept: others are considered as noise. Every block, which was not big enough to be a building, is now reconsidered: if it is neighbor of the ground, it is added to it.

Next, each building is cut into walls and roofs (figure 8). This extraction is based on the same principle as the ground extraction (details in algorithm figure 9).
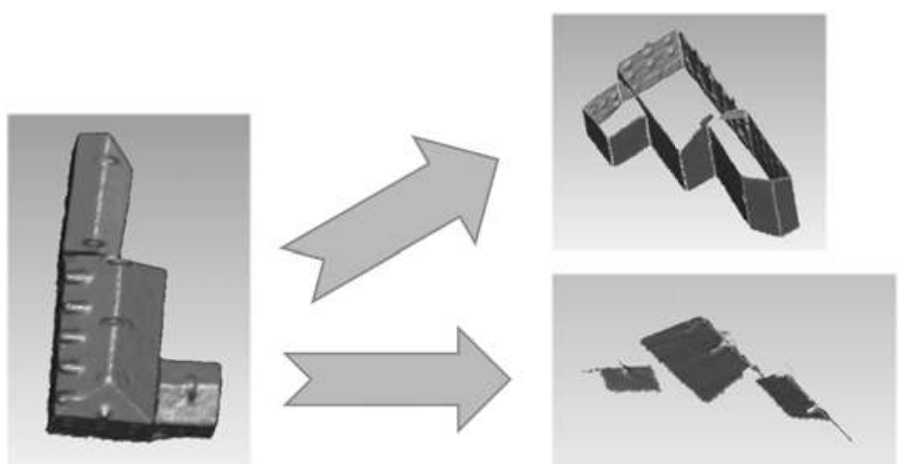

fig. 8: Wall-roof separation

Once we have obtained the walls, we can remove them from the building mesh and identify the roofs. Noisy triangles are analyzed; in function of their orientations and their neighbors, they can be added to already extracted walls and roofs.

The last part is the mesh simplification (figure 10). The building contour simplification requires to know the neighbors of each triangles and the order linking them. By order, we mean that two surfaces that are consecutive in the list of neighbors are really neighbors in the mesh (they share a triangle edge). Then for every surface we found their neighbors and sorted them.

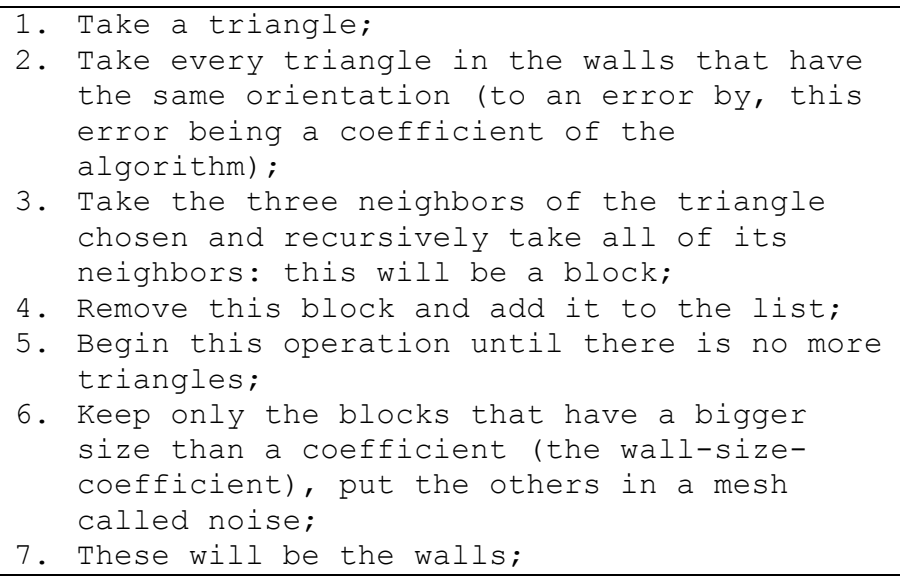

3. Take the three neighbors of the triangle chosen and recursively take all of its neighbors: this will be a block;

4. Remove this block and add it to the list;

5. Begin this operation until there is no more triangles;

6. Keep only the blocks that have a bigger size than a coefficient (the wall-sizecoefficient), put the others in a mesh called noise;

7. These will be the walls;

\section{fig. 9: mesh cut in walls algorithm}

Next, the building contour can be computed. The computation is simple: it takes the surface and two of its consecutive neighbors and then it finds the intersection edges.

We finally obtain a very simple representation of each islet that will be use in further work. Ground is not yet decimated.

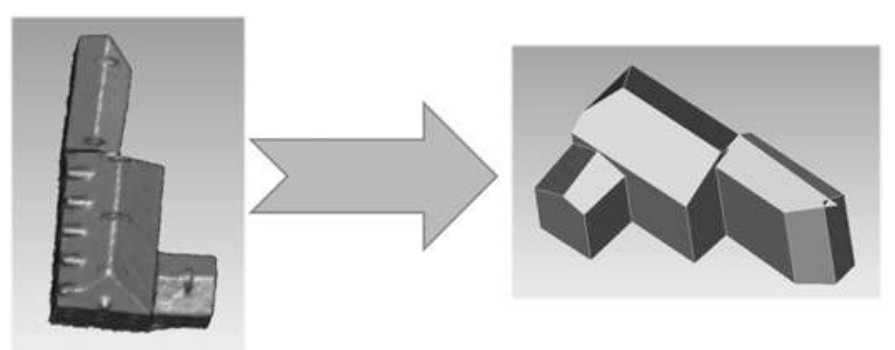

fig. 10: Mesh simplification step

\section{CONCLUSION AND PERSPECTIVES}

In this article we have presented an experience about intelligent reverse engineering. It is a first use case; 3D digitalized object is simple because it represents a mock-up of a city (with a square geometry). Semantic recognition divides the mesh into 3 elements: ground, walls and roof. Applying the same algorithm based on the orientation of the triangles, it would be possible to distinguish deeper elements as doors, windows... it is like a Russian dolls cycle; obviously we must precise that it is necessary to keep all the information we have acquired in order to optimize the semantic distinction (that means take into account the huge meshes).

Indeed, our method works at a multi-level: first applied to an islet and then to a building and then to a wall... It is a recursive algorithm that would learn how to work by itself. Due to the record of the parameters use each time, it will enrich the database and refine the parameters.

After developing the main concept of the algorithm, we have designed an open source prototype software in order to assist the user in the automatically process (figure 11). 


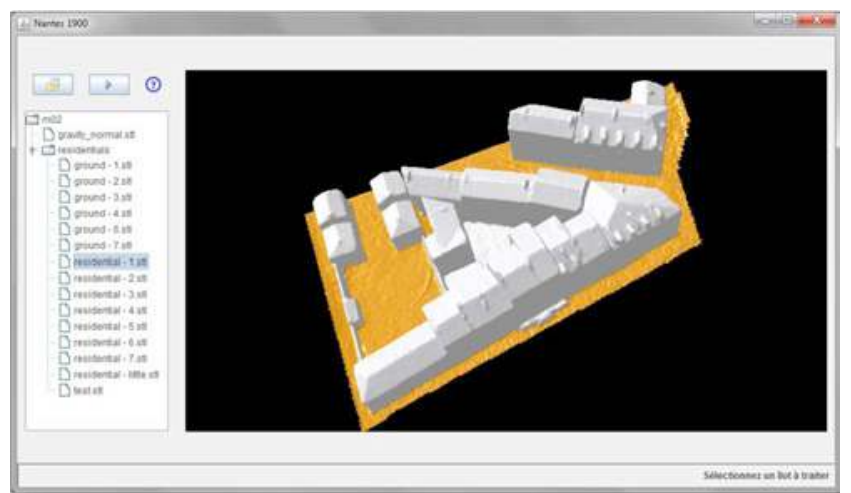

fig. 11: automatically ground selection in the software

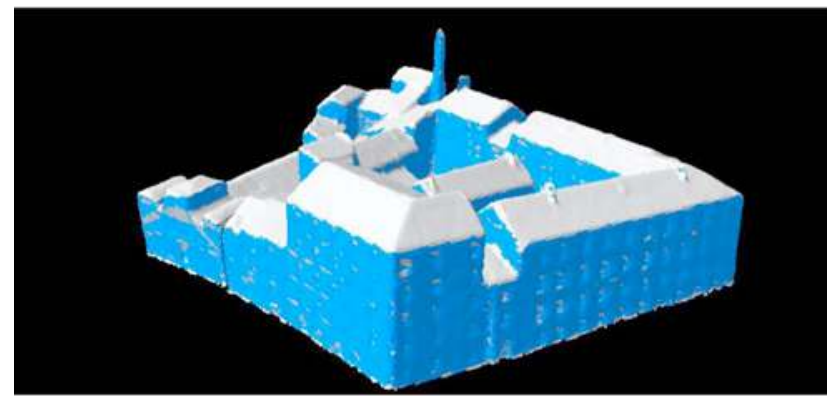

fig. 12: automatic walls selection in the software

Moreover, in the future, we aim to use our method for complex mechanical parts. Thanks to our algorithm we demonstrate that it is possible to simplify a 3D mesh but also to add semantic information for characterizing each triangle or group of triangles; in the future we would like to use the same concepts for mechanics. Now, we can link the results to a knowledge database that will contain the skeleton of the 3D object (the product conceptual model). Opening the whole 3D meshes object inside a CAD software will help the user to design new elements or to better understand a technical product. Figure 13 illustrates future process for intelligent reverse-engineering.

\section{PLURIDISCIPLINARITY AND ACKNOWLEDGMENTS}

From the museum point of view, it represents a Research \& Development aspect that is quite new in this field.

From an academic and scientific point of view, due to the fact that the project required various field of competences, it is very challenging for the researchers team to know and to choose the job required for each step of the process. Consequently, we have chosen to base Nantes1900 project upon student skills. It allows us to launch same steps with two different teams each time and at the end we compare the results and enrich our know-how.

Moreover, one of the best ways to enhance our heritage for young generation is that young people achieve the capitalization phase themselves. Until now, approximately 110 students and 10 researchers-teachers have worked on the Nantes 1900 project. Obviously it would be impossible to list all the authors in this paper so we simply would like to thanks them for their great jobs.

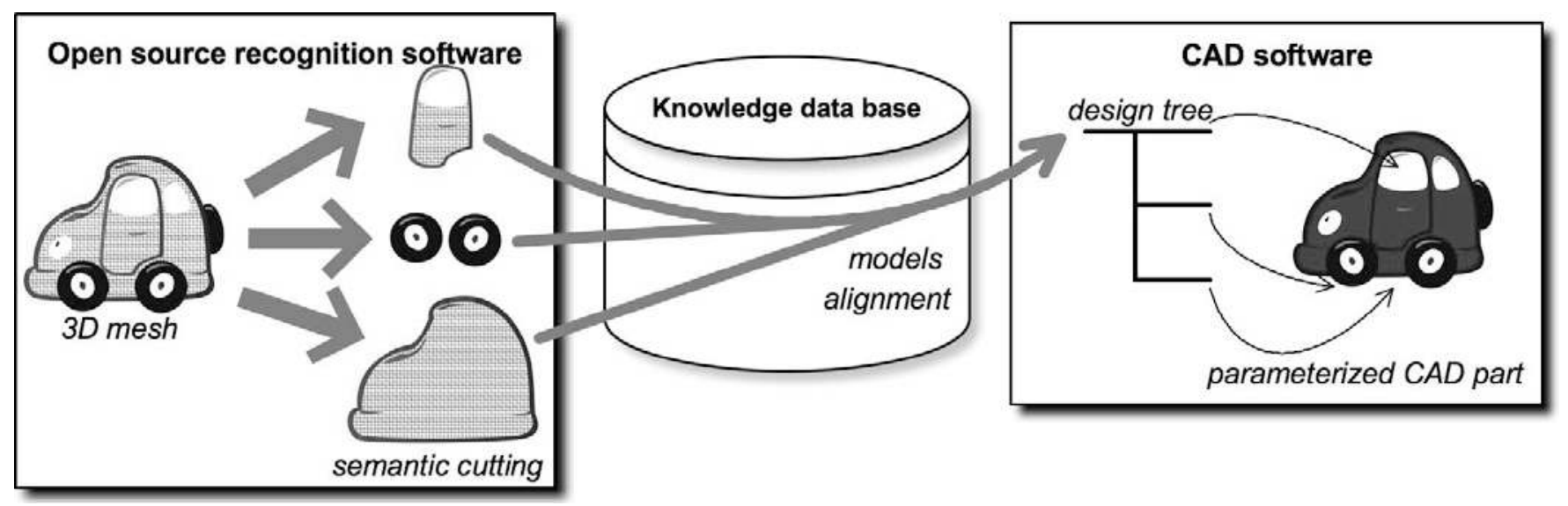

fig. 13: future process for intelligent reverse-engineering 


\section{REFERENCES}

[1] B. Guillet, L. Huron, J.-L. Kerouanton, La maquette du Port de Nantes, French congress Des villes, des ports : la mer, les hommes ( ${ }^{\circ} 124 ; 1999$; Nantes), Paris, 2001,

[2] D. Langweil, Digitalization of Prague city, last step, 2008, http://www.praguemodels.cz/

[3] C. Chevrier, K. Jacquot, J.P. Perrin, 3D modelling of a town scale model, EuroMed Conference, Limassol, Cyprus, 10 p., Nov 2010.

[4] B. Frischer, The Rome Reborn Project. How Technology is helping us to study history, OpEd, November 10, 2008. University of Virginia, 2008. [http://www.romereborn.virginia.edu/]

[5] G. Guidi, B. Frischer, et al., Virtualizing Ancient Rome: 3D Acquisition and Modeling of a Large Plaster-ofParis Model of Imperial Rome, Videometrics VIII, edited by J.Angelo Beraldin, Sabry F. El-Hakim, Armin Gruen, James S. Walton, 18-20 January 2005, San Jose, California, USA, SPIE, vol. 5665, 119-133, 2005.

[6] P. Fleury, La rome antique sur l'internet, Informatics and statistics review for human sciences, 1997.

[7] The Digital Michelangelo Project. [http://graphics.stanford.edu/projects/mich/]

$[10]$ Z. Weiss, M. Pankowski, Knowledge reengineering for reverse engineering purposes, CIRP Design Seminar Conference, Berlin, Germany, pp.421-430, 2007 - ISBN 978-3540-69819-7

[11]W. Derigent, S. Remy, E. Chapotot, G. Ris, A. Bernard, $3 D$ digitising strategy planning approach based on CAD model, Transactions of the ASME, Journal of Computing and Information Science In Engineering, vol. 7, $\mathrm{n}^{\circ}$, pp 10-19, 2007.

[12]L. De Luca, P. Véron, M. Florenzano. Reverse engineering of architectural buildings based on a hybrid modeling approach. Computers \& Graphics. Volume 30, Issue 2, Pages 160-176 Elsevier, april 2006.

[13] R. Billen, P. Blain, O. Donneau, S. Habraken, Y. Renotte, M. Van Ruymbeke. Virtual model of the city of Liège in the eighteen century - "Virtual Leodium". Proceedings of 3D Stereo MEDIA 2009, International 3D Stereo Film and Technology Festival (3D Stereo MEDIA), Liège, Belgium, 1-3 Dec. 2009. [http://hdl.handle.net/2268/34167]

[14]A. Shamir, A survey on Mesh Segmentation Techniques, COMPUTERGRAPHICS forum Volume 27 (2008), number 6 pp. 1539-1556

[15] M. Attene, S. Katz, M. Mortara, G. Patanè, M. Spagnuolo, A. Tal, Mesh segmentation - a comparative study, In: SMI '06: Proceedings of the IEEE Inter- national Conference on Shape Modeling and Applications 2006 (SMI'06), Washington, DC, USA, IEEE Computer Society (2006) 7

[16] D. P. Luebke, A developer's survey of polygonal simplification algorithms, IEEE Computer Graphics and Applications, 21(3):24-35, 2001.
[17] H. Hoppe, Progressive meshes, In Proceedings of the 23rd annual conference on Computer graphics and interactive techniques, pages 99-108. ACM Press, 1996.

[18] S. Rusinkiewicz and M. Levoy, Qsplat: a multiresolution point rendering system for large meshes, In Proceedings of the 27th annual conference on Computer graphics and interactive techniques, pages 343-352. ACM Press/Addison-Wesley Publishing Co., 2000.

[19] M. Attene, F. Robbiano, M. Spagnuolo, B. Falcidieno, Semantic Annotation of 3D Surface Meshes based on Feature Characterization, SAMT conference proceedings, 2007, 14 p.

[20] D. Porquet, Rendu en temps réel de scènes complexes, phD thesis, Université de Limoges, 2004, 162 p.

[21] M. Eck, T. DeRose, T. Duchamp, Multiresolution Analysis of Arbitrary Meshes, Technical Report, 1995, 25 p. 